\title{
A case series pilot study on the combination of 5-aminolevulinic acid and photodynamic therapy (ALA-PDT) for treatment of vitiligo*
}

\author{
YunJie Zhang ${ }^{1}$, Hui Lin ${ }^{1}$, XiaoJuan Guo ${ }^{1}$, XianBiao Zou ${ }^{1}$
}

DOI: http:/ / dx.doi.org/10.1590/abd1806-4841.20187014

\begin{abstract}
BACKGROUND: To study the effective therapeutic concentration, drug application duration, irradiation duration and irradiation dosage of 5-aminolevulinic acid photodynamic therapy (ALA-PDT) for treating vitiligo and observe its clinical efficacy. ОвјестіvE: To assess the clinical efficacy of ALA-PDT for treating vitiligo.

METHODs: ALA with different drug concentrations, drug application durations, irradiation durations and irradiation dosages were adopted to treat skin lesions caused by vitiligo to determine the effective drug concentration, drug application duration, irradiation duration and irradiation dosage for treating three vitiligo volunteers and observe the therapeutic results.

RESULTS: The clinical trial suggested that ALA at a drug concentration of $1.5 \%$, drug application duration for 3 hours, irradiation dosage of $80 \mathrm{mw} / \mathrm{cm}^{2}$ and irradiation duration for $20 \mathrm{~min}$ was effective in treating vitiligo. Under these parameters, ALA-PDT was effective to the three vitiligo volunteers, with mild pain and feeling of burning but no other adverse reaction during treatment.

STUDY LIMITATIONS: Due to the small sample size in this study for the effectiveness of PDT in treating vitiligo and the potential variations in the efficacy for treating the disease at different areas, further studies shall be conducted for confirmation. CONCLUSIONS: ALA with a drug concentration at $1.5 \%$, drug application duration for 3 hours, irradiation dosage of $80 \mathrm{mw} / \mathrm{cm}^{2}$ and irradiation duration for $20 \mathrm{~min}$ is effective in treating vitiligo. Therefore, ALA-PDT is safe and effective in treating the disease, with minor adverse events, providing a new method for treating vitiligo in the future.
\end{abstract}

Keywords: Laser therapy; Photochemotherapy; Vitiligo

\section{INTRODUCTION}

Vitiligo is an acquired local or generalized skin depigmentation disorder with a morbidity of $0.5-2 \% .{ }^{1,2}$ The disease is difficult to treat and involves a long course of therapy. Besides, patients with vitiligo receive different therapies and medicines based on their distinctive pathogenesis, triggering factors, clinical type, and conditions, with different curative effects. Our research group studied the effectiveness of the combination of 5-aminolevulinic acid and photodynamic therapy (ALA-PDT) in treating vitiligo, to observe the effective drug concentration, drug application duration, irradiation duration, and irradiation dosage. We treated three vitiligo volunteers with appropriate dosages, aiming to observe the clinical efficacy.

\section{METHODS}

The three vitiligo volunteers (two males and one female) included in this study were selected from the vitiligo patients diagnosed in our hospital's Dermatology Clinic, with age at onset between four and 28 years (mean 16 years) and course of disease between 2 and 10 years. Case 1, female, 27 years old, found a small white patch on her trunk with no obvious cause 18 years ago, which progressively grew into a large area and extended to the limbs, perineum, face and neck. According to her medical history, she was healthy, but her father had vitiligo on his hands. Case 2, male, 17 years old, found a small white patch on his abdomen with no obvious cause four years ago, which progressively grew into a larger area. He was previously healthy and had no family history of vitili-

Received 13 February 2017.

Accepted 30 May 2017.

* Work conducted at the Department of Dermatology of First Affiliated Hospital of PLA General Hospital, Beijing, China.

Financial support: Supported by Beijing Municipal Science \& Technology Commission No. Clinical study on photodynamic therapy for treatment of vitiligo; Clinical Scientific Research Supporting Fund of the General Hospital of the People's Liberation Army (special fund for the First Affiliated Hospital of the General Hospital of the People's Liberation Army) for advantageous clinical research projects: 2015FC-TSYS-3045,2014FC-TSYS-1014; National Science Fund for Distinguished Young Scholars (61308117)

Conflicts of interest: None.

1 Department of Dermatology of First Affiliated Hospital of PLA General Hospital, Beijing, China.

MAILING AdDREsS:

Xianbiao Zou

E-mail: zouxianbiao25@sina.com

C2018 by Anais Brasileiros de Dermatologia 
go. Case 3 was a male, four years old, whose parents found a small white patch on his forehead with no obvious cause two years previously, which progressively grew into an egg-size oval depigmentation patch and extended to the left eyebrow. He was previously healthy and had no family history of vitiligo.

5-aminolevulinic acid powder for external use (produced by Shanghai Shanghai Fudan-Zhangjiang Bio-Pharmaceutical Co., Ltd., specification: $118 \mathrm{mg} /$ piece) was protected from light. A LEDIB photodynamic laser therapeutic apparatus (produced by Wuhan Yage Optic and Electronic Technique Co., Ltd.) was capable of irradiating semiconductor laser with wavelength $633 \pm 10 \mathrm{~nm}$. The camera was Canon 650D.

Method for studying the effective drug concentration, drug application duration, irradiation duration, and irradiation dosage: The research project was reviewed and approved by the hospital's Ethics Committee and complies with general requirements of medical ethics. Before treatment, we communicated with the patients and their spouses, explaining the treatment process and post-treatment precautions in details. Patients and their spouses then signed informed consent after confirming their understanding of the above content. In case 1, the vitiligo skin lesion on the patient's back was selected as the treatment area (Figures 1A and 1B). The skin area was divided into 24 units. A piece of black plastic film was divided evenly into 24 grids and labeled as 24 treatment units. The plastic films were attached to the skin area and labeled with numbers to prevent shifting (Figure 1C). ALA at different concentrations was applied to the corresponding treatment units according to the concentration requirements in table 1 , and the area was covered to protect from light. Treatment was provided on the basis of preset drug application duration, irradiation duration, and irradiation dosage (Table 1 and Figure 1D). Skin reactions were observed immediately after the irradiation and at $24 \mathrm{~h}$ after treatment.

\section{Operation mode selection for PDT in vitiligo:}

Effective therapeutic concentration, drug application duration, irradiation duration, and irradiation dosage were selected on the basis of the findings from the previous experiment. According to the above parameters, PDT was used to treat three cases. After applying a certain concentration of ALA on the patients' vitiliginous area for a specific period of time, we applied PDT based on preset irradiation duration and dosage, and then observed the treatment efficacy and reactions.

\section{RESULTS}

The experiment's findings for most effective treatment concentration:

Case 1 showed immediate and obvious reactions on the dorsum (areas 9, 10, 21, and 22), plus a burning sensation during treatment and erythema after treatment (Figure 2A). At 48 hours post-treatment, areas 9, 17, 18, 21, and 22 showed post-treatment red papules around hair follicles (Figure 2B). On the basis of the reactions, the parameters for area 9 (ALA concentration 1.5\%, drug application duration $3 \mathrm{~h}$, irradiation duration $20 \mathrm{~min}$, and irradiation dosage $80 \mathrm{mw} / \mathrm{cm}^{2}$ ) were selected as the effective parameters for treating the patients, and the therapeutic results were observed.

\section{Efficacy of PDT in treating vitiligo}

According to the experiment, ALA at a concentration of $1.5 \%$, drug application duration $3 \mathrm{~h}$, irradiation dosage $80 \mathrm{mw} / \mathrm{cm}$, and irradiation duration 20min were safe and effective in treating vitiligo, with mild pain and burning sensation but no other adverse effects during treatment.

Efficacy in Case 1: Before treatment (Figure 3A). Perifollicular pigment islands were found on vitiliginous areas after the first three treatments at seven-day intervals and increased progressively
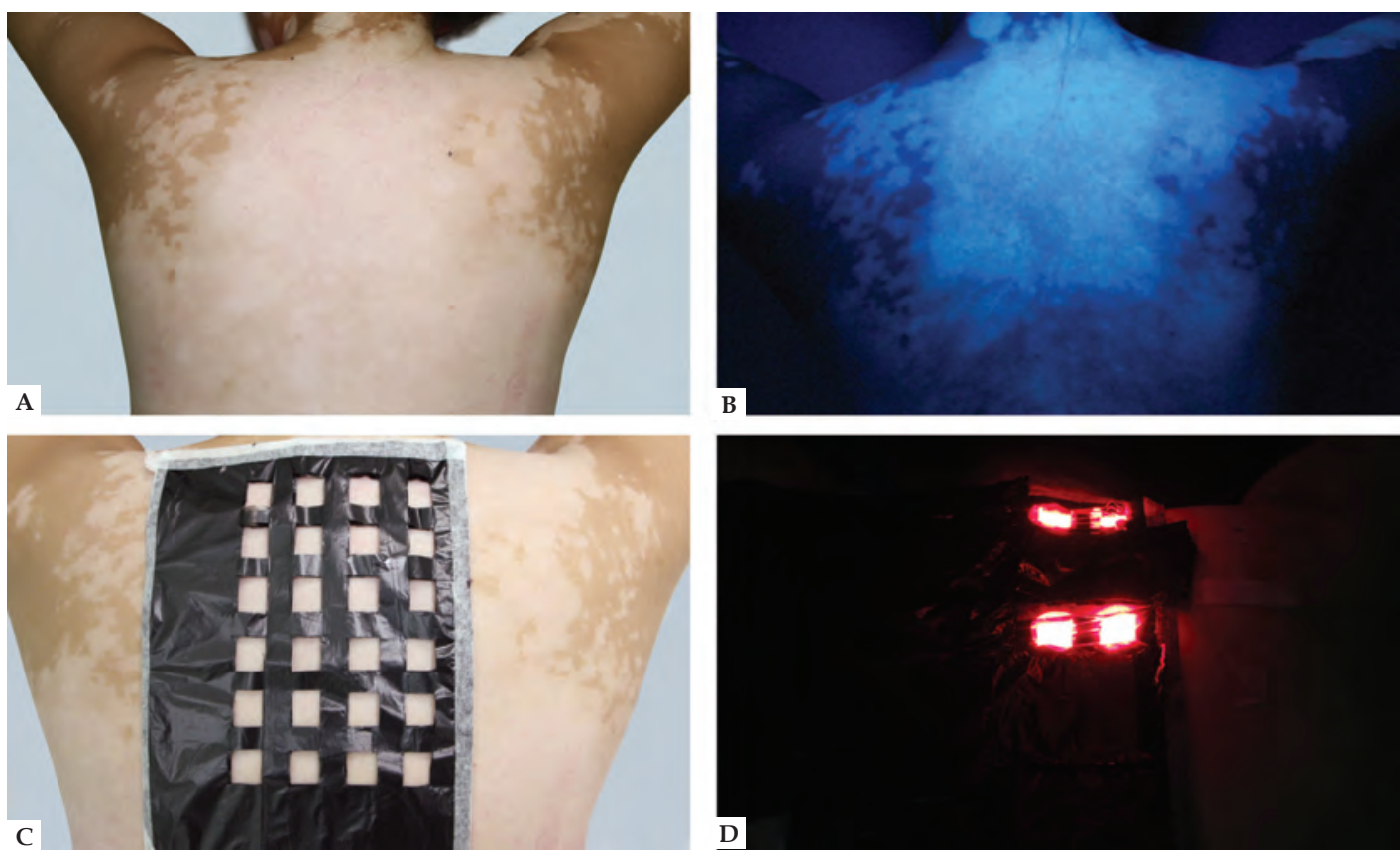

Figure 1: (A) Large white patch on the patient's back before treatment. (B) Bright white under Wood's light. (C) PDT preparations for treating vitiligo. (D) Operation of irradiation during experiment 
after seven sessions of PDT (Figures 3B and 3C). Between September 3, 2013, and November 13, 2013, the pigment islands further increased significantly, despite no PDT for 70 days (Figure 3D). From November 22, 2013, to January 20, 2014, pigment islands increased significantly on the skin in the middle of the back after six PDT treatments at 10-day intervals, with a significant effect on the vitiliginous areas of the shoulders (Figure 3E). Between January 20, 2014, and
May 4, 2014, the pigment islands shrank slightly due to discontinuance of treatment for four months (Figure 3F). From May 12, 2014, to August 4, 2014, the pigment islands increased, some forming dark spots after 14 treatments at three to five-day intervals (Figure 3G). During the 2.5-month to 1-year follow-up visit after the final treatment, no significant change was observed in the vitiliginous skin lesions (Figures 3H and 3I).

TABLE 1: Effective ALA drug concentration, drug application duration, irradiation duration, and irradiation dosage of PDT for treatment of vitiligo

Drug application duration, irradiation duration, and irradiation dosage of each treatment unit

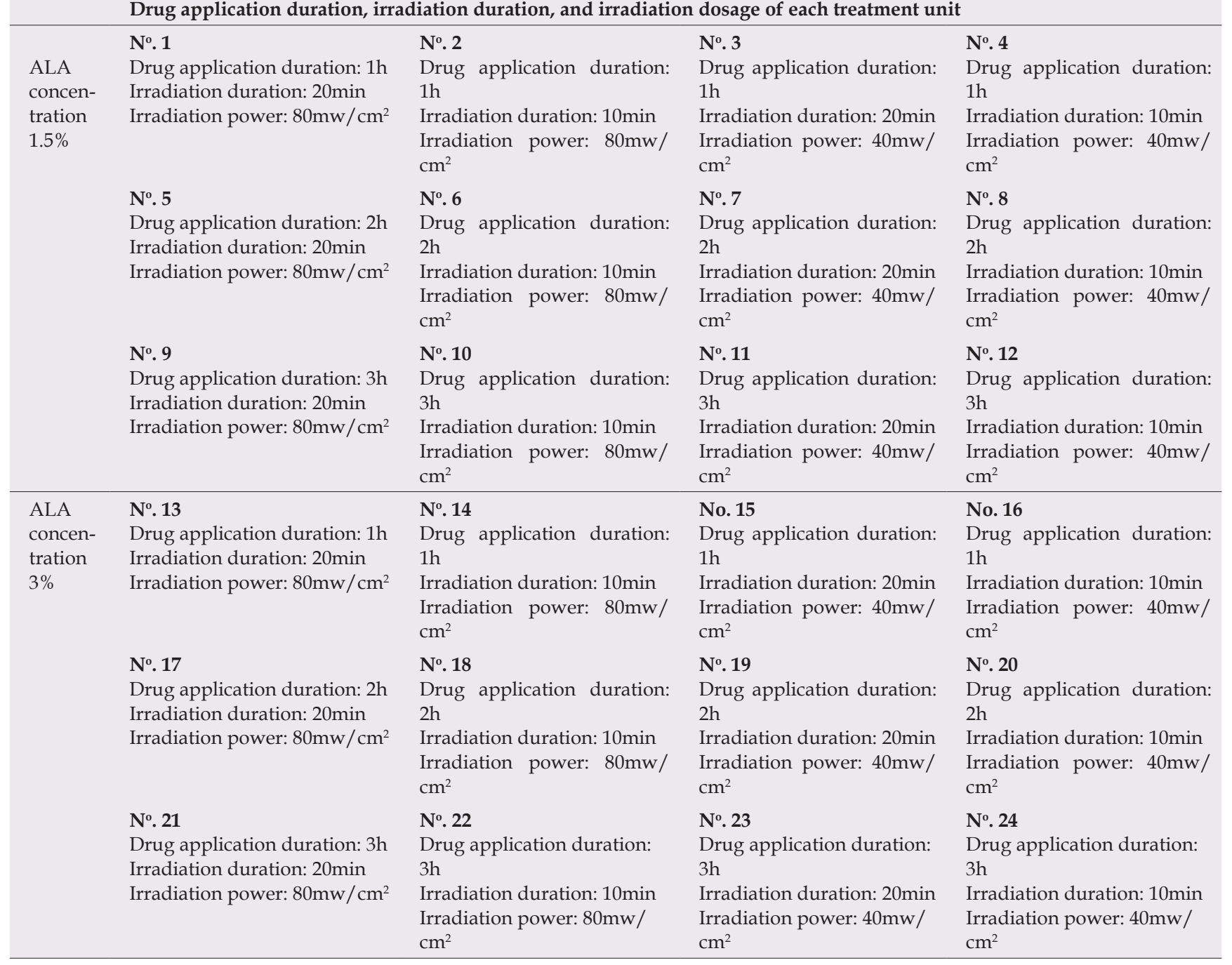
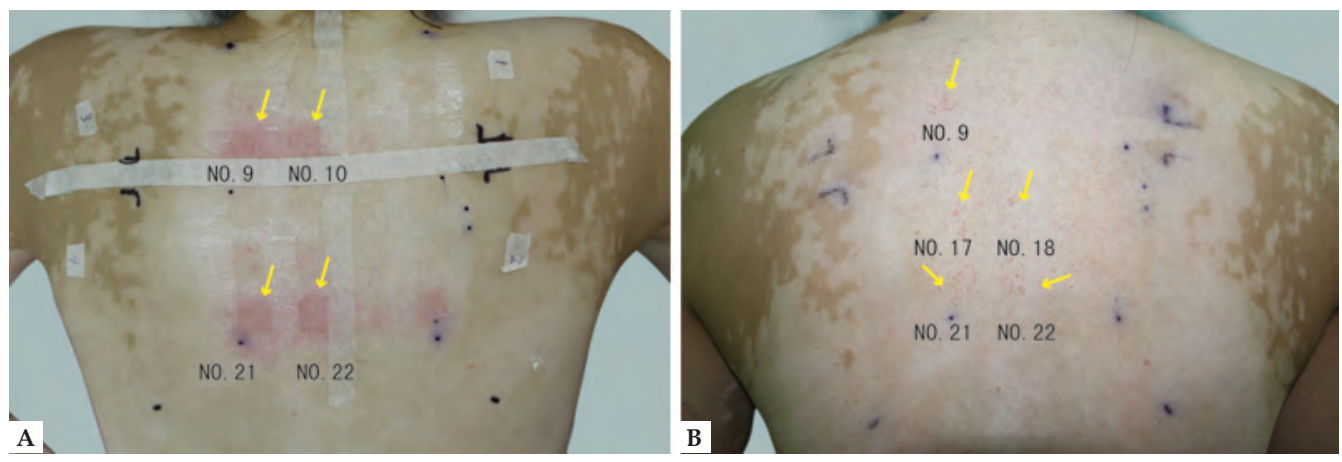

Figure 2: (A) Immediate reactions after irradiation, post-treatment erythema labeled by arrow. (B) Perifollicular red papules at $24 \mathrm{~h}$ after irradiation labeled by arrow 
Efficacy in Case 2: Before treatment (Figure 4A). Pigment islands were found around skin follicles of vitiliginous areas after the first three treatments at seven-day intervals and increased constantly after 11 PDT sessions (Figures $4 \mathrm{~B}$ and $4 \mathrm{C}$ ). After eight consecutive treatments, the pigment islands increased significantly with the number of treatments (Figure 4D). From August 28, 2015, to April 26,2016 , the pigment islands decreased partially over the course of four treatments at long intervals (74 days, 114 days, 33 days, and 15 days, respectively) in the eight months (Figure 4E). During the 75day follow-up visit after discontinuance of treatment, no significant change was observed in the vitiliginous skin lesions compared to the condition after the previous treatment (Figure $4 \mathrm{~F}$ ).

Efficacy in Case 3: Before treatment (Figure 5A). Pigment islands were seen in the vitiliginous area near the left eyebrow after three treatments (Figure 5B). After 13 PDT sessions, pigment islands were observed first on the forehead (Figure 5C). From March 21, 2016 , to June 27,2016 , after seven consecutive treatments at 10-14day intervals, the pigment islands increased significantly on the skin lesion area near the hairline, as the total number of treatments increased (Figure 5D).

\section{DISCUSSION}

Vitiligo is a common acquired progressive depigmentation skin disease with the clinical manifestation of white skin patches caused by localized or generalized depigmentation. With an overall morbidity of $0.1 \%-2 \%$, vitiligo mainly affects adolescents. ${ }^{1,2}$ Vitiligo is still difficult to treat due to the complex cause of the disease and its unclear pathogenesis. According to current studies, vitiligo is believed to correlate with autoimmunity and to be caused by multiple factors, including the patient's genetic background and the environment. Vitiligo is often accompanied by autoimmune thyroid disease, systemic lupus erythematosus, rheumatoid arthritis, alopecia areata, psoriasis, and other diseases. ${ }^{3,4} \mathrm{~V}$ Jasper G.van den Boorn et $a l .{ }^{5}$ confirmed the close correlation between T cells and vitiligo. According to some authors, compared to healthy controls and patients in the stable phase, patients in the progressive stage showed more
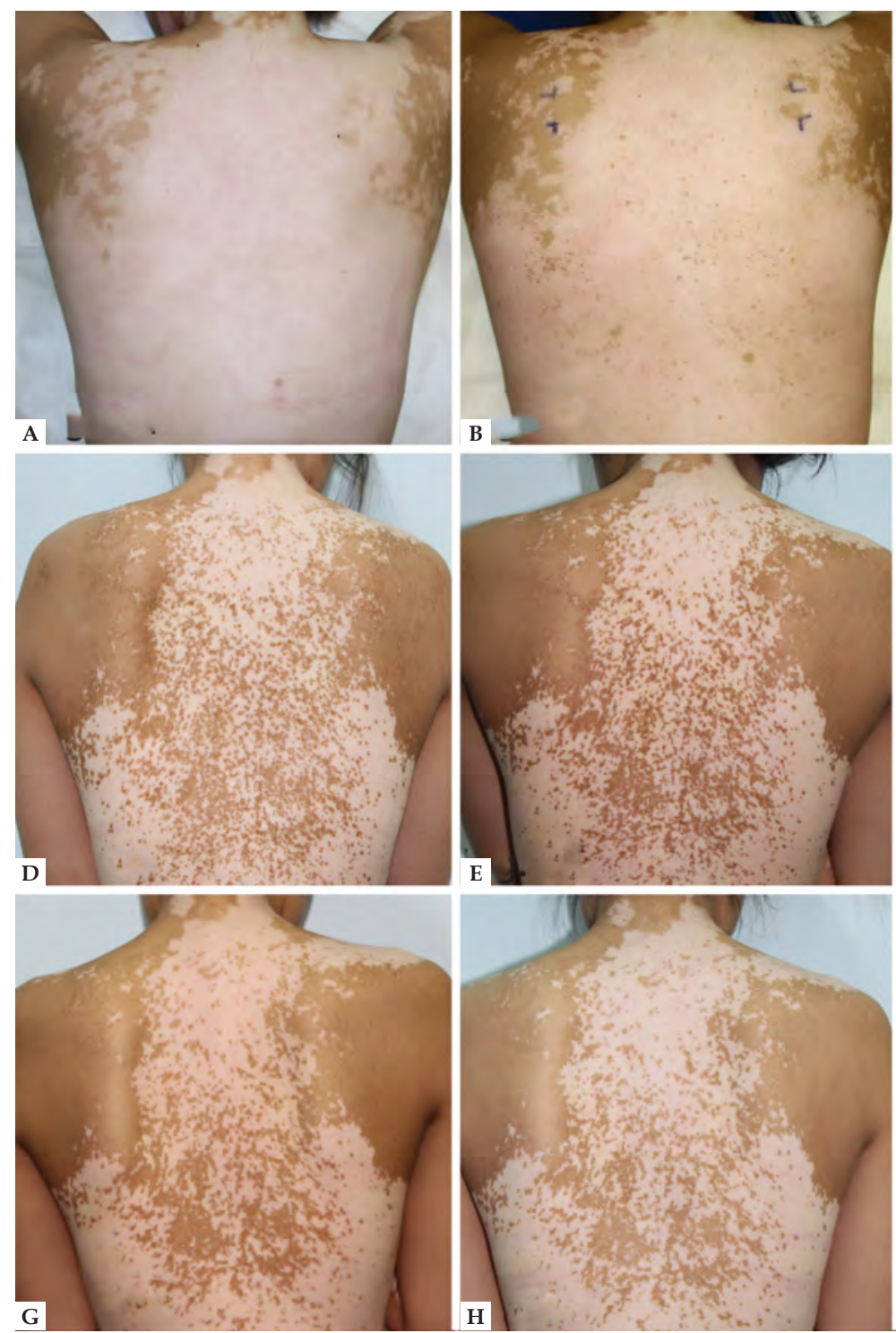
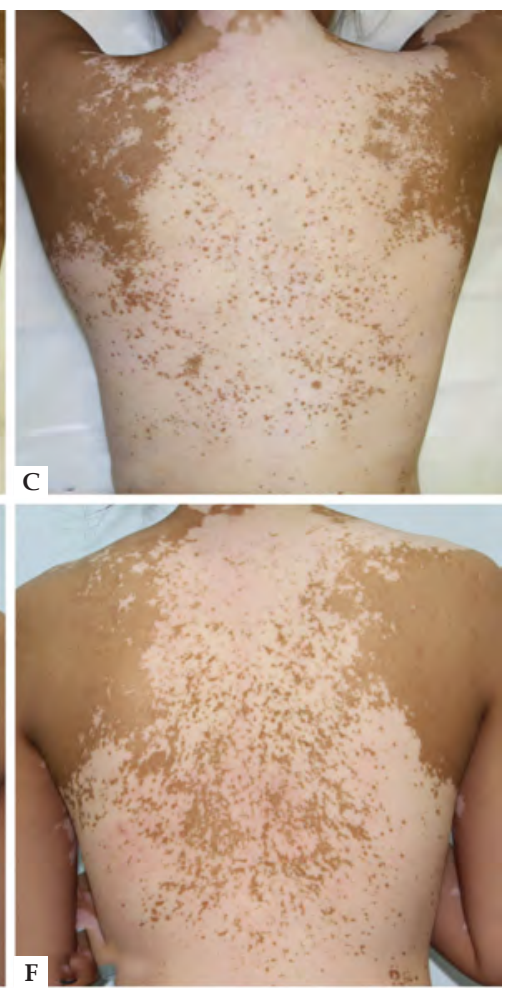

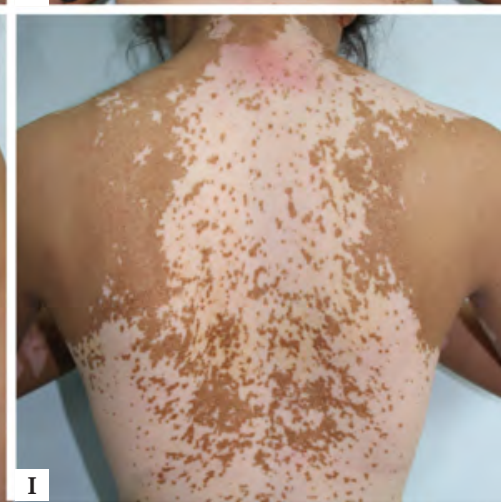

Figure 3: (A)

Case 1: Before treatment (July 10, 2013); (B). Pigment islands found on the skin of the back after three treatments (August 6, 2013); (C). Increase in pigment islands (September 3, 2013); (D). Significant increase in pigment islands (November 13 2013); (E). Significant increase in pigment islands on the skin of shoulders and in the middle of the back (January 20, 2014); (F).

Decrease in pigment islands (May 12, 2014); (G). Increase in pigment islands, dark spots formed by part of pigment islands (August 4, 2014); (H). 2.5-month follow-up visit after treatment (October 24, 2014); (I). 1-year follow-up visit after treatment (August 14, 2015). 
CD8+T cells in peripheral blood and a significantly lower proportion of CD4+/CD8+T cells. ${ }^{6}$ Lin et al. ${ }^{7}$ further confirmed that Treg cells of vitiligo patients in the progressive stage became less capable of inhibiting CD8+CLAT+ cells. In 2012, Kotobuki et al. ${ }^{8}$ found Th17 cell infiltration in the skin of most vitiligo patients. Bertolotti et al. found plasmocytoid dendritic cell ( $\mathrm{pDC}$ ) infiltration in the skin around vitiliginous lesions. $\mathrm{pDC}$ is the main type of IFN- $\alpha$-secreting cells; IFN- $\alpha$ promotes CXCL9 expression and skin homing of CXCR3+ lymphocytes by inducing MxA protein synthesis. These studies suggest a direct relationship between the human immune system and onset of vitiligo.

Currently, PDT has achieved satisfactory efficacy in treating pediatric laryngeal papilloma, condyloma acuminatum, and acne. 9-12 On the basis of the principle of PDT, photosensitization can be selectively enriched in virally infected foci after being applied locally on skin lesions. Under $635 \mathrm{~nm}$ laser irradiation, it generates singlet oxygen and other active oxides, which can damage cell membranes, mitochondria, and DNA, cause cell necrosis and apoptosis, and then kill diseased cells. ${ }^{12,13}$ In recent years, some researchers have found that PDT shows efficacy by activating local specific immunity. ${ }^{14,15}$ Brotosik et al. found that PDT resulted in apoptosis of epidermal keratinocytes and Langerhans cells. ${ }^{16}$ However, there have been no clinical studies to date on the clinical mechanism of PDT in vitiligo. It is inferred that PDT has a therapeutic effect by participating in local specific immunity. Besides, He-Ne laser adopted by PDT is a type of low-energy laser, with a laser wavelength of $633 \pm 10 \mathrm{~nm}$. It can improve melanocytes' adhesiveness on IV-type collagens, inhibit their mobility, strengthen their migration on type-I collagens, and enhance expression of $\alpha 2 \beta 1$ integrins, so as to promote melanocytes' proliferation. ${ }^{17}$ Byun et al. ${ }^{18}$ treated one case of pediatric vitiligo with He-Ne laser combined with tacrolimus ointment. After treatment for 48 weeks, most of the skin lesions showed pigment recovery. Serrano et al. ${ }^{19}$ treated six cases of vitiligo on the face, hands, and trunk by applying $2 \%$ ALA to the skin lesions four to six times at 10 -minute intervals. According to our findings, ALA-PDT was effective in treating the three cases of vitiligo. Throughout the treatment process, patients suffered mild pain and a burning sensation that could be relieved by cold compresses after treatment. After treatment, patients suffered mild desquamation with no other adverse reactions. Skin lesions were irradiated with 550-630nm laser for 4-14 minutes, with an irradiation dose of $100 \mathrm{mw} / \mathrm{cm}^{2}$ and three to four-week intervals. During the six-week follow-up visit after four treatments, four cases showed pigment recovery, with the best efficacy in the skin lesions on the chest, neck, and face. Nevertheless, M Fernández-Guarino found no pigment recovery on the face of vitiligo patients after treatment with PDT by applying MAL for 1.5 hours and irradiating under 630nm laser for 7.5min, with an irradiation dosage of $37 \mathrm{~J} / \mathrm{cm}^{2} .{ }^{20}$ Our experiment suggested that treatment unit No. 9 (applying 1.5\% ALA for 3 hours and irradiating for 20 minutes, with an irradiation dosage of $80 \mathrm{mw} / \mathrm{cm}^{2}$ ) was effective in treating vitiligo. The previous experiment for effective concentration revealed immediate and obvious reactions and erythema following PDT, and mild burning sensation during treatment (Figure 2A). After continuous observation for 48 hours, no. 17 (applying 3\% ALA for 2 hours and irradiating for 20 minutes, with an irradiation dose of $80 \mathrm{mw} /$ $\mathrm{cm}^{2}$ ), no. 18 (applying 3\% ALA for 2 hours and irradiating for 10 minutes, with an irradiation dosage of $80 \mathrm{mw} / \mathrm{cm}^{2}$ ), no. 21 (applying 3\% ALA for 3 hours and irradiating for 20 minutes, with an ir-
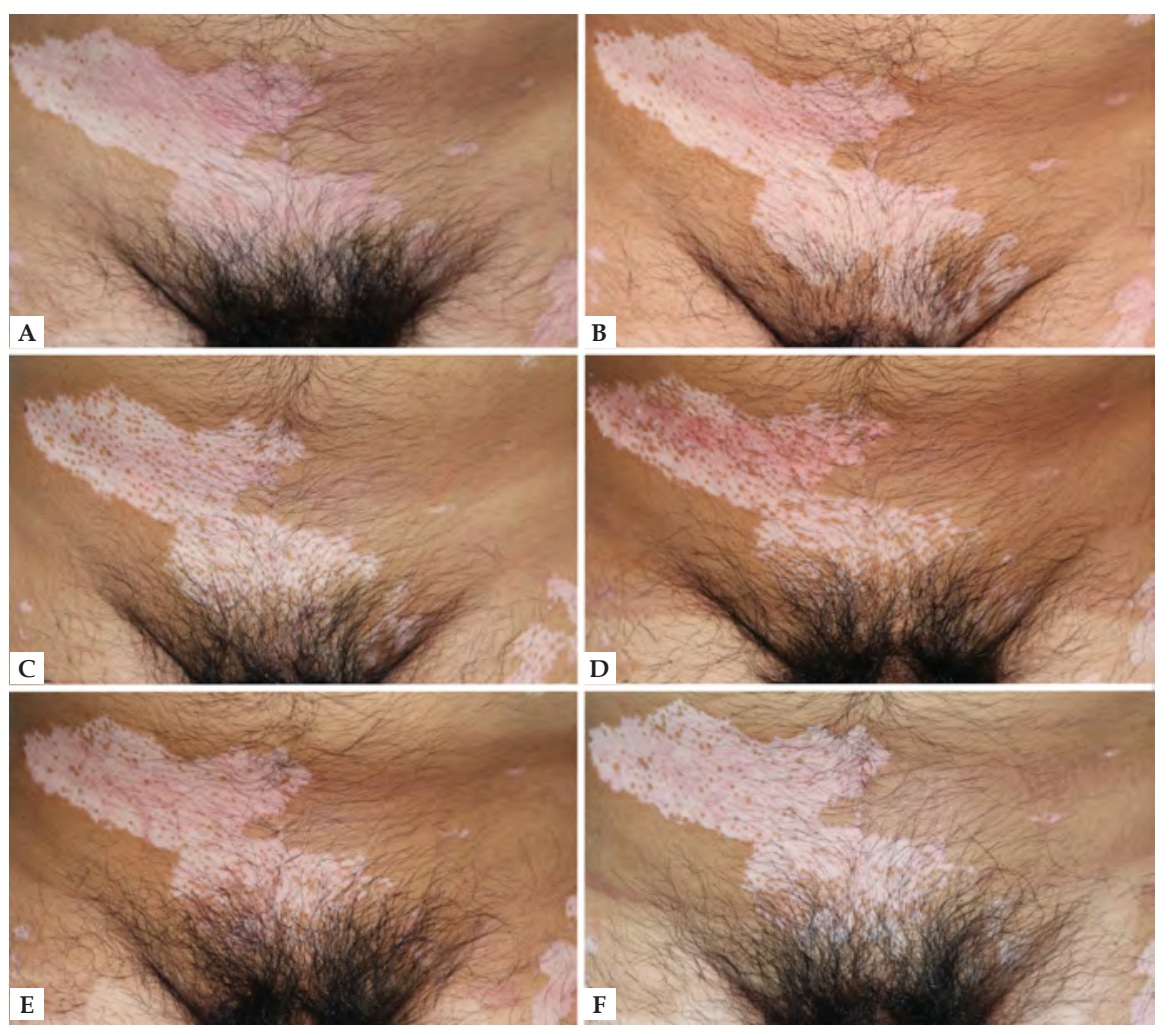

Figure 4: (A) Before treatment (June 13, 2014); (B) Increase in pigment islands after three treatments (July 11, 2014); (C) Increase in pigment islands (November 26, 2014); (D) Significant increase in pigment islands on the perineum (August 18, 2015); E: Slight decrease in pigment islands (April 26, 2016); (F) 2-month follow-up visit after final treatment (July 11, 2016) 

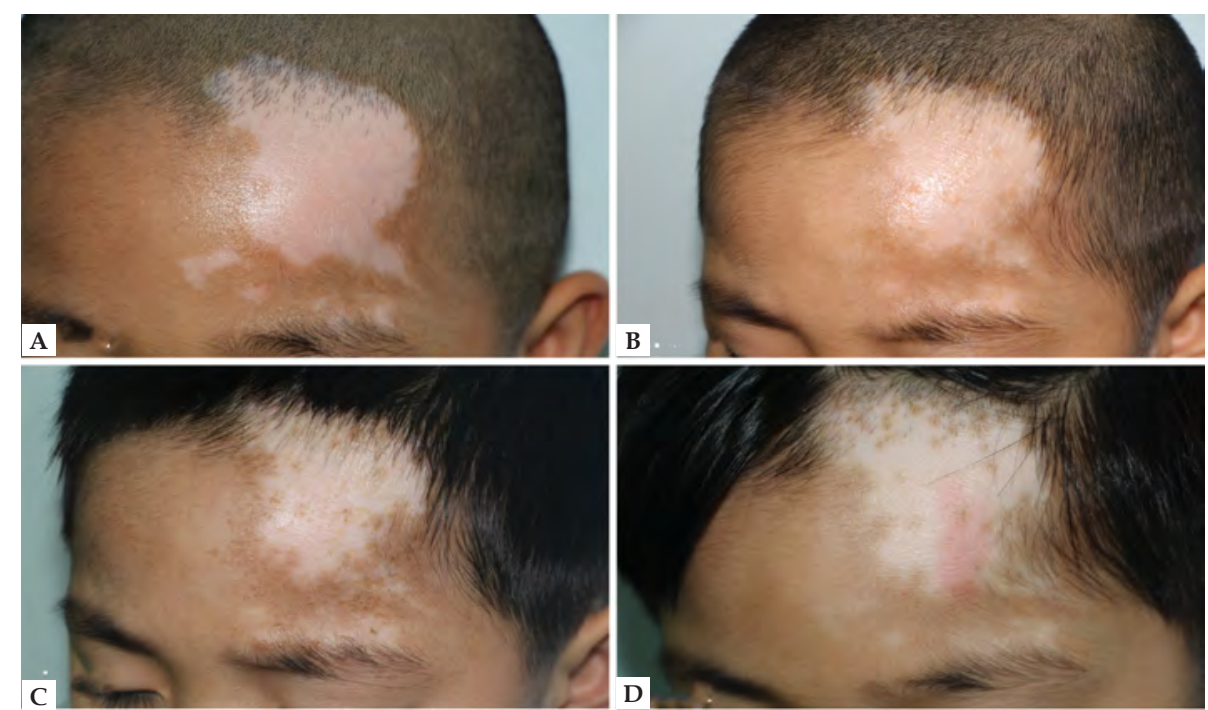

Figure 5: (A) Before treatment (August 7, 2015); (B) Pigment islands found after three treatments (October 22, 2015); (C) Pigment islands near hairline (March 7, 2016); (D) Increase in pigment islands near hairline (June 27, 2016) radiation dosage of $80 \mathrm{mw} / \mathrm{cm}^{2}$ ), and no. 22 (applying 3\% ALA for 3 hours and irradiating for 10 minutes, with an irradiation dosage of $80 \mathrm{mw} / \mathrm{cm}^{2}$ ) showed obvious reactions after treatment. Numbers $9,17,18,21$, and 22 showed post-treatment perifollicular reactions (Figure 2B). As for therapeutic safety, we selected the parameters from low-concentration treatment unit no. 9 (applying 1.5\% ALA for 3 hours and irradiating for 20 minutes at an irradiation dosage of $80 \mathrm{mw} / \mathrm{cm}^{2}$ ) as the therapeutic dosage for the three cases. The experiment also showed that higher irradiation can cause more obvious reactions. Among the 24 treatment units, with the same parameters, cases with $80 \mathrm{mw} / \mathrm{cm}^{2}$ irradiation showed more obvious reactions than those with $40 \mathrm{mw} / \mathrm{cm}^{2}$.

ALA at a concentration at $1.5 \%$, drug application for $3 \mathrm{~h}$, irradiation dosage of $80 \mathrm{mw} / \mathrm{cm}^{2}$, and irradiation duration for $20 \mathrm{mi}-$ nutes was adopted to treat the three vitiligo patients. Perifollicular pigment islands were seen in vitiliginous areas after the first three treatments at seven-day intervals and increased with the number of treatments (Figures 3B, 4B, and 5B). In case 1, from July 12, 2013, to January 20, 2014, pigment islands increased significantly with the number of treatments at seven to 10-day intervals. From May 12 to August 4, 2014, pigment islands increased slightly after treatments at three to five-day intervals, indicating unsatisfactory efficacy due to the short intervals (Figures 3F and 3G); From January 20, 2014, to May 12, 2014, pigment islands decreased partially due to discontinuance of treatment. In case 2, from June 13 to October 15, 2014, pigment islands increased significantly with the number of treatments at seven to 10-day intervals. From November 26, 2014, to August 10,2015 , pigment islands increased only slightly after treatments at
20 to 30-day intervals, indicating unsatisfactory efficacy due to the long intervals (Figures 4C and 4D). From August 18, 2015, to April 26,2016 , the pigment islands shrank partially after only four treatments in the eight months. In case 3, only a few pigment islands were seen in the vitiliginous area near the left eyebrow after the first three treatments, and melanin began to appear on the forehead after 11 PDT sessions (Figure 5D). From March 21 to June 27, 2016, as the number of treatments increased, pigment islands increased significantly after seven treatments at seven to 10-day intervals (Figure 5D). According to the efficacy in the three cases, ALA at $1.5 \%$, drug application duration of 3 hours, irradiation dosage of $80 \mathrm{mw} /$ $\mathrm{cm}^{2}$, and irradiation duration of 20 minutes proved to be effective in treating vitiligo. Due to the reactions after the first three treatments, the treatments were made at one to two-week intervals, achieving satisfactory efficacy, and the pigment islands increased with the number of treatments. Pigment islands decreased during prolonged discontinuance of treatment, which impacted efficacy.

However, due to the small sample size in this study on the effectiveness of ALA-PDT in vitiligo and the potential variations in the efficacy for treating vitiligo on different areas, further studies will be conducted for confirmation.

\section{CONCLUSION}

According to the findings, ALA-PDT was effective in treating three cases of vitiligo. During treatment, patients suffered mild pain and a burning sensation, relieved by cold compresses after treatment; After treatment, patients suffered mild desquamation, with no other adverse reactions. $\square$ 


\section{REFERENCES}

1. Krüger $\mathrm{C}$, Schallreuter $\mathrm{KU}$. A review of the worldwide prevalence of vitiligo in children/adolescents and adults. Int J Dermatol. 2012;51:1206-12.

2. Zhang XJ, Chen JJ, Liu JB. The genetic concept of vitiligo. J Dermatol Sci. 2005;39:137-46.

3. Kemp EH, Waterman EA, Weetman AP. Autoimmune aspects of vitiligo. Autoimmunity. 2001;34:65-77.

4. Kroon MW, Vrijman C, Chandeck C, Wind BS, Wolkerstorfer A, Luiten RM, et al. High Prevalence of Autoimmune Thyroiditis in Children and Adolescents with Vitiligo. Horm Res Paediatr. 2013;79:137-44.

5. van den Boorn JG, Konijnenberg D, Dellemijn TA, van der Veen JP, Bos JD, Melief $\mathrm{CJ}$, et al. Autoimmune destruction of skin melanocytes by perilesional T cells from vitiligo patients. J Invest Dermatol. 2009;129:2220-32.

6. Dwivedi M, Laddha NC, Arora P, Marfatia YS, Begum R. Decreased regulatory T-cells and CD4 $(+) / C D 8(+)$ ratio correlate with disease onset and progression in patients with generalized vitiligo. Pigment Cell Melanoma Res. 2013;26:586-91.

7. Lin M, Zhang BX, Shen N, Dong XJ, Zhang C, Qi XY, et al. Regulatory T cells from active non-segmental vitligo exhibit lower suppressive ability on CD8+CLA+ T cells. Eur J Dermatol. 2014;24:676-82.

8. Kotobuki Y, Tanemura A, Yang L, Itoi S, Wataya-Kaneda M, Murota H, et al. Dysregulation of melanocyte function by Th17-related cytokines: significance of Th17 cell infiltration in autoimmune vitiligo vulgaris. Pigment Cell Melanoma Res. 2012;25:219-30

9. Wang XL, Wang HW, Wang HS, Xu SZ, Liao KH, Hillemanns P. Topica 5-aminolaevulinic Acid-photodynamic Therapy for the Treatment of Urethral Condylomata Acuminata. Br J Dermatol. 2004;151:880-5.

10. Keyal U, Bhatta AK, Wang XL. Photodynamic therapy for the treatment of different severity of acne: A systematic review. Photodiagnosis Photodyn Ther. 2016;14:191-9.
11. Yang YG, Zou XB, Zhao H, Zhang YJ, Li HJ.Photodynamic therapy of condyloma acuminata in pregnant women. Chin Med J (Engl). 2012;125:2925-8.

12. Zhang $Y$, Yang $Y$, Zou X, Huang Z. 5-Aminolevulinic Acid Photodynamic Therapy combined with CO2 Laser Therapy in treatment of laryngeal papilloma: Case report. Photodiagnosis Photodyn Ther. 2016;14:131-3.

13. Zhang Y, Yang Y, Zou X. Efficacy of 5-Aminolevulinic acid Photodynamic Therapy in treatment of nasal inverted papilloma. Photodiagnosis Photodyn Ther. 2013;10:549-51.

14. Kubiak M, tysenko L, Gerber H, Nowak R. Cell reactions and immune responses to photodynamic therapy in oncology. Postepy Hig Med Dosw (Online). 2016;70:735-42.

15. Kleinovink JW, van Driel PB, Snoeks TJ, Prokopi N, Fransen MF, Cruz LJ, et al. Combination of photodynamic therapy and specific immunotherapy efficiently eradicates established tumors. Clin Cancer Res. 2016;22:1459-68.

16. Bartosik J, Stender IM, Kobayasi T, Agren MS.. Ultrastructural alteration of tape-stripped normal human skin after photodynamic therapy. Eur J Dermatol. 2004;14:91-5.

17. Lan CC, Wu CS, Chiou MH, Chiang TY, Yu HS. Low-energy helium-neon laser induces melanocyte proliferation via interaction with type IV collagen: visible light as a therapeutic option for vitiligo. Br J Dermatol. 2009;161:273-80.

18. Byun JW, Babitha S, Kim EK, Shin J. A successful helium neon laser and topical tacrolimus combination therapy in one child with vitiligo. Dermatol Ther. 2015;28:333-5.

19. Serrano G, Lorente M, Reyes M, Millán F, Lloret A, Melendez J, et al. Photodynamic therapy with low-strength ALA, repeated applications and short contact periods (40-60minutes) in acne, photoaging and vitilligo. J Drugs Dermatol. 2009;8:562-8.

20. Fernández-Guarino $\mathrm{M}$, Harto $\mathrm{A}$, Jaén P. Photodynamic therapy does not induce repigment in three cases of facial vitiligo. J Eur Acad Dermatol Venereol. 2008;22:1498-500.

\section{AUTHORS CONTRIBUTION}

Yunjie Zhang

iD ORCID 0000-0003-0866-5819

Elaboration and writing of the manuscript; Obtaining, analyzing and interpreting the data; Effective participation in research orientation

Hui Lin

D ORCID 0000-0001-5239-568X

Effective participation in research orientation

Xiaojuan Guo
Effective participation in research orientation
Xianbiao Zou
Approval of the final version of the manuscript; Conception and planning of the study

How to cite this article: Zhang YJ, Lin H, Guo XJ, Zou XB. A case series pilot study on the combination of 5-aminolevulinic acid and photodynamic therapy (ALA-PDT) for treatment of vitiligo. An Bras Dermatol. 2018;93(4):539-45. 